Benchmarks

\title{
Global analysis of RNA oxidation in Saccharomyces cerevisiae
}

Anastasia McKinlay ${ }^{1,3}$, Wayne Gerard ${ }^{1}$, and Stanley Fields ${ }^{1,2,3}$

${ }^{1}$ Department of Genome Sciences, University of Washington, Seattle, WA, USA, ${ }^{2}$ Department of Medicine, University of Washington, Seattle, WA, USA, and ${ }^{3}$ Howard Hughes Medical Institute, University of Washington, Seattle, WA, USA.

BioTechniques 52:109-111 (February 2012) doi 10.2144/000113801

Keywords: yeast; Saccharomyces cerevisiae; RNA modification; RNA oxidation; 8-OHG modification; free radicals; hydrogen peroxide treatment

Oxidative RNA damage has been linked to loss of RNA function and to the development of many human age-related diseases. Consequently, a need exists for methods to identify and quantify the extent of RNA oxidation on a genome-wide basis. We developed such a method by combining affinity selection of mRNA containing 8-hydroxyguanine with high throughput DNA sequencing. We demonstrate that this assay is suitable for detecting differences in the extent of oxidation between RNA transcripts. We applied this method to the yeast Saccharomyces cerevisiae grown under physiological conditions and in response to hydrogen peroxide, and detected significantly oxidized RNA transcripts.

Formation of reactive oxygen species (ROS) is unavoidable in living cells. During normal physiological processes (such as respiration), $0.1 \%-2 \%$ of molecular oxygen $\left(\mathrm{O}_{2}\right)$ is converted to the superoxide anion $\left(\mathrm{O}_{2}\right)(1)$. However, under oxidative stress, levels of ROS increase, resulting in damage to proteins, lipids, DNA and RNA. Cellular RNA is highly prone to oxidative damage (2). RNA oxidation has been linked to the loss or alteration of RNA function, and the accumulation of dysfunctional RNA might contribute to the development of cancers and age-related degenerative diseases (2-4).

Several approaches have been used to measure RNA oxidative damage (4). Some are based on detection of 8-hydroxyguanine (8-OHG), the most abundant and deleterious modification, by high performance liquid chromatography (HPLC) equipped with electrochemical and UV detectors (5), or HPLC coupled with electrospray tandem mass spectrometry (6). Other approaches use monoclonal antibodies against 8-OHG in immunohistochemical or Northwestern methods to assess RNA oxidation in tissues and subpopulations of RNAs $(7,8)$. For example, using Alzheimer patients' brain tissues, Shan et al. (8) isolated oxidized RNA via affinity purification with antibodies against 8-OHG and cloned the corresponding $\mathrm{cDNA}$ into a plasmid. Sequencing of a few clones revealed oxidative damage-susceptible RNAs, some of which had been linked to the pathogenesis of Alzheimer disease.

Here we describe a genome-wide assay to identify oxidized RNA transcripts that relies on the affinity purification of oxidized RNA on anti-8-OHG antibody-coupled beads followed by high-throughput Illumina sequencing of a DNA library prepared from this RNA. In parallel, we assessed total RNA abundance by construction and sequencing of DNA libraries from RNA that had not undergone the affinity purification for 8-OHG. Total RNA from Saccharomyces cerevisiae strain BY4742 (9) was extracted with hot phenol (Invitrogen, Carlsbad, CA, USA) and treated with Turbo DNase I (Ambion, Austin, TX, USA), and then partially depleted of rRNA by an mRNA purification kit (Invitrogen). An aliquot of the mRNA-enriched total RNA was used for total RNA library construction (see below). Oxidized RNA was precipitated from total RNA with anti8-OHG antibody (QED Bioscience, Inc., San Diego, CA, USA) coupled to Protein G (Invitrogen). Binding ( $75 \mu \mathrm{g}$ total RNA to $25 \mu \mathrm{L}$ protein $\mathrm{G}$ coupled to $2.5 \mu \mathrm{g} 8$-OHG antibody) was performed in PBS buffer in the presence of SUPERase-In (Ambion) for $1 \mathrm{~h}$ at room temperature with rotation. Beads were first washed two times with 500 $\mu \mathrm{L}$ PBS buffer with $0.04 \%$ NP40 (Invitrogen), transferred to a new tube and then washed twice again with $500 \mu \mathrm{L}$ PBS buffer with $0.04 \%$ NP40 for $10 \mathrm{~min}$, followed by RNA extraction with $200 \mu \mathrm{L}$ phenol chloroform-isoamyl alcohol (Invitrogen). Approximately $2 \mu \mathrm{g}$ of mRNA-enriched total RNA was immunoprecipitated by antibody-coupled beads. To estimate background binding, we subjected the same amount of mRNA-enriched total RNA to

Table 1. Sequencing data acquisition and mapping statistics

\begin{tabular}{|c|c|c|c|c|c|}
\hline Library & $\begin{array}{c}\text { Reads } \\
\text { acquired }\end{array}$ & $\begin{array}{c}\text { Reads } \\
\text { mapped }\end{array}$ & $\begin{array}{c}\text { Percent } \\
\text { mapped }\end{array}$ & $\begin{array}{c}\text { Unique, non- } \\
\text { rRNA reads }\end{array}$ & $\begin{array}{c}\text { Unique, non- } \\
\text { rRNA reads (\%) }\end{array}$ \\
\hline Oxidzed RNA & $64,581,760$ & $57,775,230$ & $89.46 \%$ & $19,008,947$ & $32.90 \%$ \\
\hline Total RNA & $68,738,128$ & $61,787,617$ & $89.89 \%$ & $19,678,020$ & $31.84 \%$ \\
\hline Oxidized RNA $+\mathrm{H}_{2} \mathrm{O}_{2}$ & $75,142,091$ & $65,930,518$ & $87.74 \%$ & $17,592,399$ & $26.68 \%$ \\
\hline Total RNA $+\mathrm{H}_{2} \mathrm{O}_{2}$ & $83,896,685$ & $72,330,257$ & $86.21 \%$ & $23,539,861$ & $32.54 \%$ \\
\hline
\end{tabular}

To define mappable bases we identified all unique 36 base sequences from both strands of the reference assembly. We defined mappable bases as all positions covered by these sequences. 
the immunoprecipitation procedure using beads alone. In contrast to elution from the antibody-coupled beads, which yielded $\sim 400 \mathrm{ng}$ of RNA, the elutions from the negative control beads yielded $\sim 100 \mathrm{ng}$ or less of lower quality RNA, suggesting that $<5 \%$ of the RNA applied to control beads bound. Approximately 50 ng of oxidized RNA and mRNA-enriched total RNA were fragmented (Ambion Fragmentation kit, Ambion) and used for strand-specific Illumina sequencing library preparation (10).

Strand-specific Illumina sequencing libraries were constructed from RNA isolated from two independent cultures grown in yeast extract-peptone dextrose (YPD) medium at $30^{\circ} \mathrm{C}$ to exponential growth phase $\left(\mathrm{OD}_{600}=0.5-0.6\right)$. The cultures had either been left untreated, or were treated prior to harvest for $15 \mathrm{~min}$ with $0.5 \mathrm{mM}$ hydrogen peroxide. After aligning reads to the genome, we filtered out low quality alignments and rRNA sequences. The reproducibility between the two biological replicates of immunoprecipitated oxidized RNA libraries, and between the replicates of the total RNA libraries, was robust (Pearson's $r^{2}>0.99$ ). Therefore, we merged reads from biological replicates and obtained four data sets: total
Table 2. Significantly expressed and significantly expressed oxidized transcripts

\begin{tabular}{|c|c|c|}
\hline Library & $\begin{array}{c}\text { Significantly } \\
\text { expressed }\end{array}$ & $\begin{array}{c}\text { Significantly } \\
\text { expressed \& oxidized }\end{array}$ \\
\hline Oxidzed RNA & $2,921(46.40 \%)$ & $892(14.16 \%)$ \\
\hline Total RNA & $3,454(54.80 \%)$ & \\
\hline Oxidized RNA $+\mathrm{H}_{2} \mathrm{O}_{2}$ & $2,444(38.79 \%)$ & $1,222(19.40 \%)$ \\
\hline Total RNA $+\mathrm{H}_{2} \mathrm{O}_{2}$ & $3,451(54.78 \%)$ & \\
\hline
\end{tabular}

RNA from the untreated and hydrogen peroxide-treated yeast, and oxidized RNA from the untreated and hydrogen peroxidetreated yeast (Table 1 ).

Of the 6,300 yeast transcript models, 40\%-55\% were significantly expressed in either total or oxidized RNA libraries, as measured against the background from intergenic regions using a Poisson distribution $(P<0.01)$ (Table 2$)$. For each significantly expressed yeast transcript in each data set, we calculated an RNA oxidation index as the ratio of normalized reads in the oxidized RNA to those in the total RNA. We defined significantly oxidized transcripts as transcripts with read density in an oxidized RNA sample significantly higher than in the corresponding total RNA sample using a Poisson exact test (Q-value cutoff of 0.001 ). This analysis revealed that $14 \%$ and $19 \%$ of the yeast transcripts were significantly oxidized in the untreated sample and hydrogen peroxide-treated sample, respectively (Table 2 ).

Approximately 91\% (815 of 892 ) of the transcripts found to be oxidized in untreated yeast were also identified from yeast subjected to oxidative stress, suggesting that these transcripts might possess features that make them vulnerable to physiological levels of free radicals. Treatment with hydrogen peroxide resulted in an additional 407 transcripts classified as significantly oxidized. Approximately $9 \%$ (77 of 892) of the transcripts were detected as oxidized only under physiological growth conditions. Some of these transcripts may represent nonspecifically bound RNAs, or they may carry the 8-OHG modification to regulate a step in RNA metabolism, such as stability, splicing, transport, or translational efficiency.

\section{BioTechniques Subscriptions}

\section{Subscriptions are FREE to qualified subscribers!}

BioTechniques has provided readers with innovative laboratory methods and techniques for nearly 30 years. From early descriptions of DNA extraction procedures and PCR methods to recent thought-provoking reviews and perspectives, BioTechniques articles are timely, topical, and will have an immediate impact on your work in the lab.

\section{Publishing in 2012:}

Focus Sections:

- The Modern Lab

- The Rise of High-throughput Technology

- Tools to Engineer the Genome
Tech News Features:

Advances in Cell Biology

Microfluidics

Cell Culture

Metagenomics

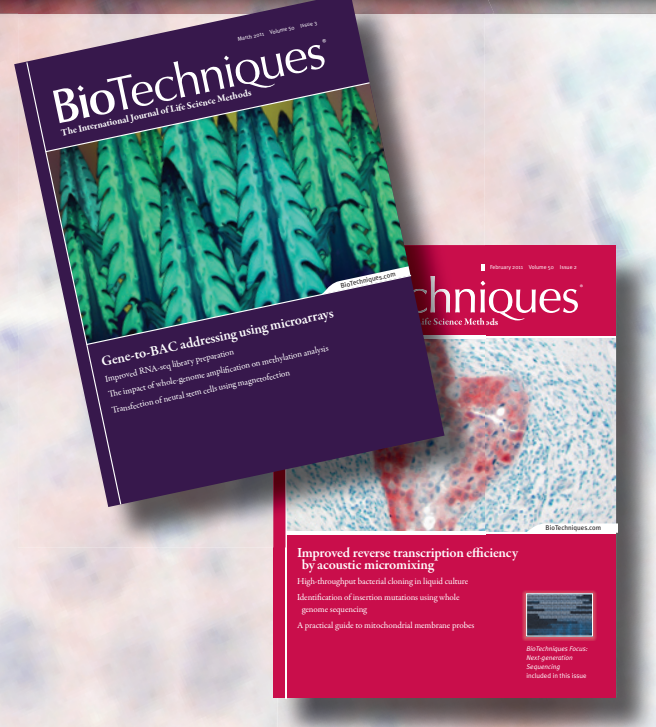

Subscribe, Update, or Renew Your Subscription Today! 
We examined the effect of oxidative stress on the overlapped set of 815 yeast transcripts identified as oxidized. We predicted that these transcripts overall should be more oxidized in response to $\mathrm{H}_{2} \mathrm{O}_{2}$ treatment. For each transcript, we calculated a ratio of its oxidative index in the $\mathrm{H}_{2} \mathrm{O}_{2}$-treated sample to that in the untreated sample. This analysis revealed that 594 out of the $815(73 \%)$ transcripts had a ratio $>1$ (with all but one transcript having a ratio below 2), suggesting that these are sensitive to oxidative stress, whereas for 221 (27\%) of the transcripts, the ratio fell below 1 .

We explored the correlation between the extent of RNA oxidation and features of the RNA. To examine the correlation with RNA folding, we determined RNA folding energies after normalizing the minimum free energy of RNA folding $(\triangle G)(11)$ by transcript length $(\triangle G /$ length) (12). This analysis revealed a modest correlation (Spearman $\left.\rho^{2}<-0.372\right)$ between RNA oxidation index and $\triangle \mathrm{G} /$ length, indicating that highly structured RNAs are less oxidized by ROS. Susceptibility to oxidative damage may be affected by RNA subcellular localization, because mRNA targeted to mitochondria likely encounters higher levels of ROS. We compared the RNA oxidation index with the mitochondria location index (MLR) calculated for 3106 yeast transcripts based on their association with free and mitochondrion-bound polysomes (13). We observed modest correlation between these parameters (Spearman $\left.\rho^{2}>0.41\right)$. We observed no significant correlation between RNA oxidation and either RNA abundance, transcript length, or ribosomal density (14).

In summary, we developed a highthroughput method that is suitable to identify oxidized RNA species on a genome-wide scale, and applied this method to $S$. cerevisiae. Similar studies performed on RNA isolated from tissues of patients affected by neurodegenerative diseases might shed light on the role of RNA oxidation in these diseases.

\section{Acknowledgments}

We thank Charlie Lee and Jay Shendure for help with DNA sequencing. This work was supported by P41 RR11823 from the NIH.S.F. is an investigator of the Howard Hughes Medical Institute.

\section{Competing interests}

The authors declare no competing interests.

\section{References}

1. Brand, M.D. 2005. The efficiency and plasticity of mitochondrial energy transduction. Biochem. Soc. Trans. 33:897-904.

2. Li, Z., J. Wu, and C.J. Deleo. 2006. RNA damage and surveillance under oxidative stress. IUBMB Life 58:581-588.

3. Radak, Z. and I. Boldogh. 2010. 8-Oxo-7,8dihydroguanine: links to gene expression, aging, and defense against oxidative stress. Free Radic. Biol. Med. 49:587-596.

4. Wurtmann, E.J. and S.L. Wolin. 2009. RNA under attack: cellular handling of RNA damage. Crit. Rev. Biochem. Mol. Biol. 44:34-49.

5. Shen, Z., W. Wu, and S.L. Hazen. 2000. Activated leukocytes oxidatively damage DNA, RNA, and the nucleotide pool through halidedependent formation of hydroxyl radical. Biochemistry 39:5474-5482.

6. Weimann, A., D. Belling, and H.E. Poulsen. 2002. Quantification of 8-oxo-guanine and guanine as the nucleobase, nucleoside and deoxynucleoside forms in human urine by high-performance liquid chromatography-electrospray tandem mass spectrometry. Nucleic Acids Res. 30(2):E7.

7. Nunomura, A., G. Perry, M.A. Pappolla, R. Wade, K. Hirai, S. Chiba, and M.A. Smith. 1999. RNA oxidation is a prominent feature of vulnerable neurons in Alzheimer's disease. J. Neurosci. 19:1959-1964.

8. Shan, X., H. Tashiro, and C.L. Lin. 2003. The identification and characterization of oxidized RNAs in Alzheimer's disease. J. Neurosci. 23:4913-4921.

9. Brachmann, C.B., A. Davies, G.J. Cost, E. Caputo, J. Li, P. Hieter, and J.D. Boeke. 1998. Designer deletion strains derived from Saccharomyces cerevisiae S288C: a useful set of strains and plasmids for PCR-mediated gene disruption and other applications. Yeast 14:115-132.

10. Parkhomchuk, D., T. Borodina, V. Amstislavskiy, M. Banaru, L. Hallen, S. Krobitsch, H. Lehrach, and A. Soldatov. 2009. Transcriptome analysis by strand-specific sequencing of complementary DNA. Nucleic Acids Res. 37:e123.

11. Hofacker, I.L. 2003. Vienna RNA secondary structure server. Nucleic Acids Res. 31:3429-3431.

12. Freyhult, E., P.P. Gardner, and V. Moulton. 2005. A comparison of RNA folding measures. BMC Bioinformatics 6:241.

13. Marc, P., A. Margeot, F. Devaux, C. Blugeon, M. Corral-Debrinski, and C. Jacq. 2002. Genome-wide analysis of mRNAs targeted to yeast mitochondria. EMBO Rep. 3:159-164.

14. Ingolia, N.T., S. Ghaemmaghami, J.R. Newman, and J.S. Weissman. 2009. Genome-wide analysis in vivo of translation with nucleotide resolution using ribosome profiling. Science 324:218-223.

Received 05 September 2011; accepted 22 December 2011.

AddresscorrespondencetoStanley Fields, University ofWashington, Seattle, WA 98195.Email: fields@u. washington.edu

To purchase reprints of this article, contact: biotechniques@fosterprinting.com
TAKE ACTION AGAINST CONTAMINATION WITH QMI ${ }^{\triangleright}$ SAFE-SEPTUM

Sample, Inoculate Or Add Nutrients To Your Bioreactor Aseptically !

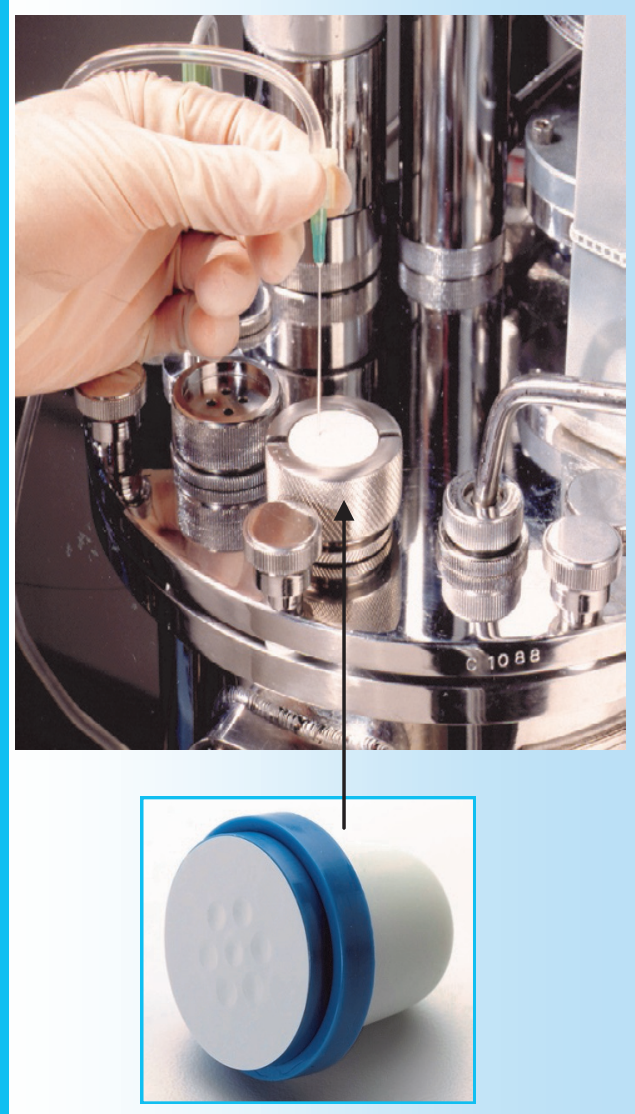

The QMI Safe-Septum is:

\section{Aseptic}

Pressure \& Temperature Safe Pre-Sterilized Easy To Retrofit Validated

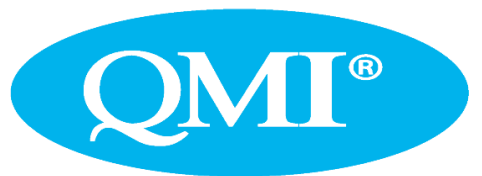

651-501-2337

Email: info@qmisystems.com 